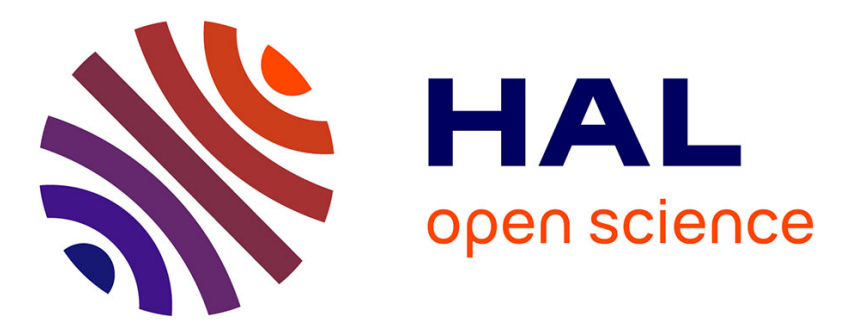

\title{
Modeling heat transfer in dilute two-phase flows using the Mesoscopic Eulerian Formalism
}

Jérôme Dombard, Benoît Leveugle, Laurent Selle, Julien Reveillon, Thierry

Poinsot, Yves d'Angelo

\section{- To cite this version:}

Jérôme Dombard, Benoît Leveugle, Laurent Selle, Julien Reveillon, Thierry Poinsot, et al.. Modeling heat transfer in dilute two-phase flows using the Mesoscopic Eulerian Formalism. International Journal of Heat and Mass Transfer, 2012, vol. 55, pp.1486-1495. 10.1016/j.ijheatmasstransfer.2011.10.050 . hal-00811401

\section{HAL Id: hal-00811401 \\ https://hal.science/hal-00811401}

Submitted on 10 Apr 2013

HAL is a multi-disciplinary open access archive for the deposit and dissemination of scientific research documents, whether they are published or not. The documents may come from teaching and research institutions in France or abroad, or from public or private research centers.
L'archive ouverte pluridisciplinaire HAL, est destinée au dépôt et à la diffusion de documents scientifiques de niveau recherche, publiés ou non, émanant des établissements d'enseignement et de recherche français ou étrangers, des laboratoires publics ou privés. 


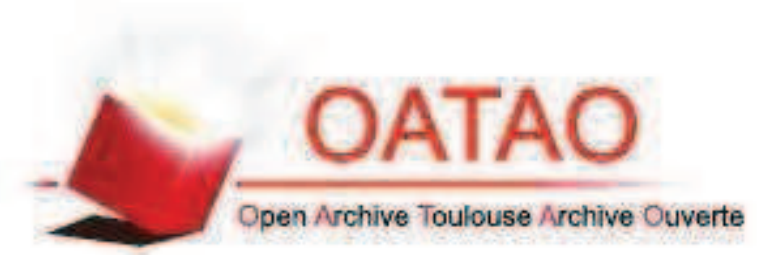

\section{Open Archive TOULOUSE Archive Ouverte (OATAO)}

OATAO is an open access repository that collects the work of Toulouse researchers and makes it freely available over the web where possible.

This is an author-deposited version published in : http://oatao.univ-toulouse.fr/ Eprints ID : 8565

To link to this article : DOI: 10.1016/j.ijheatmasstransfer.2011.10.050

URL : http://dx.doi.org/10.1016/j.ijheatmasstransfer.2011.10.050

To cite this version :

Dombard, Jérôme and Leveugle, Benoît and Selle, Laurent and Reveillon, Julien and Poinsot, Thierry and D'Angelo, Yves Modeling heat transfer in dilute two-phase flows using the Mesoscopic Eulerian Formalism. (2012) International Journal of Heat and Mass Transfer, vol. 55 ( $\mathrm{n}^{\circ}$ 5-6). pp. 1486-1495. ISSN 0017-9310

Any correspondence concerning this service should be sent to the repository administrator: staff-oatao@listes.diff.inp-toulouse.fr 


\title{
Modeling heat transfer in dilute two-phase flows using the Mesoscopic Eulerian Formalism
}

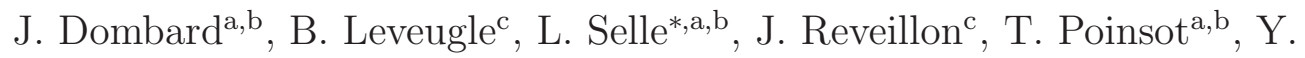 \\ D'angeloc \\ ${ }^{a}$ Université de Toulouse; INPT, UPS; IMFT (Institut de Mécanique des Fluides de \\ Toulouse); Allée Camille Soula, F-31400 Toulouse, France \\ ${ }^{b}$ CNRS; IMFT; F-31400 Toulouse, France \\ ${ }^{c}$ University of Rouen, CORIA UMR-CNRS, 6614, Avenue de l'Université,76801 Saint \\ Etienne du Rouvray, France
}

\begin{abstract}
In dilute two-phase flows, accurate prediction of the temperature of the dispersed phase can be of paramount importance. Indeed, processes such as evaporation or chemical reactions are strongly non-linear functions of heat transfer between the carrier and dispersed phases. This study is devoted to the validation of an Eulerian description of the dispersed phase -the Mesoscopic Eulerian Formalism (MEF) - in the case of non-isothermal flows. Direct numerical simulations using the MEF are compared to a reference Lagrangian simulation for a two-dimensional non-isothermal turbulent jet laden with solid particles. The objectives of this paper are (1) to study the influence of the thermal inertia of particles on their temperature distribution and (2) conduct an a posteriori validation of the MEF, which was recently extended to non-isothermal flows. The focus is on the influence of additional terms in the MEF governing equations, namely heat fluxes arising from the
\end{abstract}

\footnotetext{
*corresponding author.

Email address: laurent.selle@imft.fr (L. Selle)
} 
Random Uncorrelated Motion (RUM). Results show that mean and rms of particle temperature are strongly dependent of the thermal Stokes number. The mean temperature is satisfactorily predicted by the MEF, comparing to the Lagrangian reference. Under the conditions of the present study, the RUM heat fluxes have a marginal influence on the mean particle temperature. However, a significant impact was observed on the magnitude of particle temperature fluctuations. Neglecting the RUM heat fluxes leads to erroneous results while the Lagrangian statistics are recovered when it is accounted for in the regimes of low to moderate thermal Stokes number.

Key words:

Particle-laden flows, Mesoscopic Eulerian Formalism, Eulerian-Eulerian approach, Heat transfer

\section{Introduction}

A variety of industrial devices involve two-phase flows and many of them are non-isothermal. In combustion chambers, for example, fuel is injected in liquid state at a relatively low temperature into a hot, turbulent flow. Due to hydrodynamic forces, the liquid is atomized into droplets. The subsequent evaporation of the droplets' cloud is driven by heat exchange between the carrier and the dispersed phase. Moreover, temperature fluctuations may have a strong impact on the local evaporated fuel mass fraction as evaporation is a non-linear phenomenon. The resulting fluctuations of local equivalence ratio are known to have a negative impact on ignition, flame propagation or even combustion instabilities $[1,2,3]$. An accurate description of heat transfer to the dispersed phase is therefore necessary. 
Very few studies have directly tackled the issue of particle temperature dispersion, which is mainly due to the lack of experimental data of nonisothermal two-phase flows [4]. Recent experimental techniques -such as rainbow thermometry- seem promising but further improvements are required [5]. An alternative is to use Direct Numerical Simulations (DNS): a few studies of non-isothermal academic configurations, coupled with lagrangian tracking of particles, have been carried out. The mechanism of twophase heat and turbulent transport by particles was investigated in an decaying isotropic turbulence with an imposed temperature gradient in the fluid [6]: it was found that the particle temperature fluctuation and velocity are well correlated in the direction of the imposed temperature gradient. Jaberi et al. [7] investigated the effects of the particle dynamical response time, $\tau_{p}$, the Prandtl number, Pr, the Reynolds number, Re and mass-loading ratio, $r_{m l}$, on the statistics of particle temperature in a non-isothermal isotropic turbulence with stationary velocity and temperature fluctuations. They showed that particle temperature fluctuations decrease as $\tau_{p}$, Pr, Re and $r_{m l}$ increase. An extension of this work [8] showed that the response of particle temperature is different when the fluid and particle temperature decay in isotropic turbulence. In this case, the variance of the fluid and particle temperatures increase when the magnitude of $r_{m l} \times \operatorname{Pr}$ increases. Shotorban et al. [9] studied the dispersed-phase temperature statistics in particle-ladden turbulent homogeneous shear flow in the presence of mean temperature gradient. They found that the particle temperature variance increases when the ratio of specific heat increases.

The Eulerian-Lagrangian (EL) approach is as a powerful tool to under- 
stand and simulate two-phase flows in academic configurations. However, the lagrangian tracking of individual particles for the simulation of a realistic industrial configuration is still beyond reach because of the large number of droplets. An alternative is to model the dispersed phase as a continuum, like the carrier: this approach is called Eulerian-Eulerian (EE). The equilibrium Eulerian approach, recently extended to non-isothermal flows gives promising results [10] but is adapted only to particles with sufficiently small dynamical and thermal inertia. The statistical approach proposed by Février et al. [11], called the Mesoscopic Eulerian formalism (MEF), is able to reproduce local and instantaneous properties of particles embedded in a turbulent fluid flow [12]. The cornerstone of the MEF is the partitioning of the particle velocity field into two contributions: a continuous, self-coherent velocity shared by all particles called the mesoscopic field and a spatially uncorrelated contribution referred to as Random Uncorrelated Motion (RUM). This formalism showed its ability to simulate correctly turbulent two-phase flows in a complex geometry [13] and was recently extended to non-isothermal conditions [14]. A priori tests in a non-isothermal droplet-laden turbulent planar jet [15] show the ability of this approach to describe an evaporating dispersed phase interacting with a turbulent flow. The objective of the present work is twofold:

1. study the influence of the particles' thermal inertia in a configuration representative of a spray injection in a combustion chamber.

2. and propose an a posteriori validation of the MEF extended to nonisothermal flows.

The organization of the paper is as follows: the two solvers and modeling 
equations are described in Sec. 2; the configuration and boundary conditions are then presented in Sec. 3; finally the results are presented in Sec. 4 with detailed validation of the dynamics and temperature of the dispersed phase.

\section{Description of the solvers and modeling equations}

These simulations are carried out by two different codes developed at CERFACS and CORIA:

- a dilatable low-Mach solver (Asphodele - CORIA) with lagrangian tracking of individual particles.

- a compressible code (AVBP - CERFACS), where the MEF has been implemented.

\subsection{Carrier phase flow solvers}

Numerical methods used for the carrier-phase flow solvers have been already described in the litterature $[16,17,18]$ and are only summarized here. Boundary conditions are treated in Sec. 3.2. AVBP solves the compressible Navier-Stokes equations. A third-order in time and space, finite-element scheme TTGC [19] is used for the carrier and dispersed phase. Asphodele is a DNS structured low-Mach solver. It uses a fourth-order finite-difference scheme for the gas and a third-order explicit Runge-Kutta scheme with a minimal data storage method [20] for both carrier and dispersed phases. A third-order interpolation is employed for the determination of gaseous phase properties at the location of a particle. 


\subsection{Eulerian/Lagrangian formulation}

As described by Reeks [21], it is possible to take into account many forces to characterize the particle dynamics. However, because of the high density ratio between dispersed and gas phases, only the drag force, which is prevalent, has been retained. Additionally, several usual assumptions have been made: some of them are given in the following, but details may be found in a reference paper of Sirignano [22]. First, the spray is supposed dispersed and each particle is unaware of the existence of the others. Any internal heterogeneity or particle rotation is neglected and an infinite heat conduction coefficient is assumed in the particle. As a consequence, the particle temperature remains uniform but evolves with time. As a first approach and because of the dispersed nature of the flow, a one-way coupling has been considered. By denoting $\mathbf{V}_{p}$ and $\mathbf{X}_{p}$ the velocity and position vectors of a particle, respectively, the following relations are used to track particles throughout the computational domain:

$$
\begin{aligned}
\frac{d \mathbf{V}_{p}}{d t} & =\frac{1}{\tau_{p}}\left(\mathbf{U}\left(\mathbf{X}_{p}, t\right)-\mathbf{V}_{p}\right) \\
\frac{d \mathbf{X}_{p}}{d t} & =\mathbf{V}_{p}
\end{aligned}
$$

The vector $\mathbf{U}\left(\mathbf{X}_{p}, t\right)$ represents the gas velocity at the particle $\mathbf{X}_{p}$. The right hand side term of Eq. (1) stands for a drag force applied to the particle and $\tau_{p}$ is the kinetic relaxation time:

$$
\tau_{p}=\frac{\rho_{p} d_{p}^{2}}{18 \mu_{f}}
$$


where $d_{p}$ is the particle diameter, $\rho_{p}$ is the dispersed phase density and $\mu_{f}$ is the gas viscosity. The heating of each particle is caracterized through

$$
\frac{d T_{p}}{d t}=\frac{1}{\tau_{\theta}}\left(T\left(\mathbf{X}_{d}\right)-T_{p}\right)
$$

where the characteristic relaxation time $\tau_{\theta}$ is defined as:

$$
\tau_{\theta}=\frac{P_{r}}{12} \frac{C_{p}}{C_{f}} \frac{\rho_{p} d_{p}^{2}}{\mu}=\frac{3}{2} \operatorname{Pr} \alpha \tau_{p}
$$

where the gas and particle constant heat capacities are denoted $C_{f}$ and $C_{p}$, respectively. $\operatorname{Pr}$ is the Prandtl number. The particle-to-fluid heat capacity ratio is $\alpha=C_{p} / C_{f}$.

\subsection{Eulerian/Eulerian formulation: the Mesoscopic Eulerian Formalism}

\subsubsection{General presentation}

The MEF was originally presented by Février et al. [11]: using Direct Numerical Simulations, they observed that two arbitrarily-close particles may have drastically different velocities. In other words, the ratio of the two-point correlation between particle velocities and the particle kinetic energy does not reach unity when the distance goes to zero (c.f. their Fig. 3). Based on this observation, the cornerstone of the $\mathrm{MEF}$ is a statistical-average operator, $\left\langle\bullet \mid \mathcal{H}_{f}\right\rangle$ that corresponds to the average over all particle realizations for a fixed carrier-fluid realization $\mathcal{H}_{f}$. This operator splits the particle velocity, $u_{p}$, in two contributions: a continuous, self-coherent velocity, $\tilde{u}_{p}=\left\langle u_{p} \mid \mathcal{H}_{f}\right\rangle$, shared by all particles called the mesoscopic field and a spatially uncorrelated contribution, $\delta u_{p}$, referred to as Random Uncorrelated Motion. One has

$$
u_{p}(t)=\tilde{u}_{p}\left(x_{p}(t), t\right)+\delta u_{p}(t),
$$


where $x_{p}(t)$ is the position of the particle at time $t$. Similarly, one can decompose the particle temperature $T_{p}$ into its mesoscopic, $\widetilde{T}_{p}$, and uncorrelated, $\delta T_{p}$, components:

$$
T_{p}(t)=\widetilde{T}_{p}\left(x_{p}(t), t\right)+\delta T_{p}(t) .
$$

From the perspective of particle dynamics, it can be simply said that the mesoscopic velocity and temperature are related to the coupling with the carrier phase, through drag and heat transfer, and that the RUM is caused by the inertia of the particles. Indeed, because of inertial effects, two particles may get to neighboring locations with different trajectories and therefore different properties (velocity, temperature, etc.). Consequently, for particle dynamics, the ratio of the inertial and viscous time scales acting on the particles is central for the evaluation of the relative importance of the mesoscopic and uncorrelated contributions. This ratio is the Stokes number, St, defined as

$$
\mathrm{St}=\frac{\tau_{p}}{\tau_{f}}
$$

where $\tau_{p}$ is the particle relaxation time and $\tau_{f}$ a time scale typical of the carrier phase. Using the particle thermal time scale $\tau_{\theta}$ a thermal Stokes number, $\mathrm{St}_{\theta}$, may be also defined as

$$
\mathrm{St}_{\theta}=\frac{\tau_{\theta}}{\tau_{f}}
$$

The dynamical and thermal particle relaxation times, $\tau_{p}$ and $\tau_{\theta}$, have been defined in Eq. 3 and Eq. 5, respectively. However, the choice of a characteristic time, $\tau_{f}$, for the carrier fluid can be ambiguous depending on the configuration [23]. For the present configuration (c.f. Sec. 3), the momentum thickness at the inlet boundary condition, $\delta_{\theta}$, is chosen as the reference 
length and the maximum of the rms of the inlet velocity, $u_{f}^{\prime m a x}$, is chosen as the reference velocity, leading to:

$$
\tau_{f}=\frac{\delta_{\theta}}{u_{f}^{\prime \max }} .
$$

\subsubsection{Governing equations}

The set of Eulerian equations for a non-isothermal dilute particle flow in the mesoscopic formalism was derived by Masi [14]:

$$
\begin{aligned}
\frac{\partial \tilde{n}_{p}}{\partial t}+\frac{\partial \tilde{n}_{p} \tilde{u}_{p, j}}{\partial x_{j}} & =0 \\
\frac{\partial \tilde{n}_{p} \tilde{u}_{p, i}}{\partial t}+\frac{\partial \tilde{n}_{p} \tilde{u}_{p, i} \tilde{u}_{p, j}}{\partial x_{j}} & =-\frac{\tilde{n}_{p}}{\tau_{p}}\left(\tilde{u}_{p, i}-u_{f, i}\right)-\frac{\partial \tilde{n}_{p} \delta R_{p, i j}}{\partial x_{j}}, \\
\frac{\partial \tilde{n}_{p} C_{p} \widetilde{T}_{p}}{\partial t}+\frac{\partial \tilde{n}_{p} C_{p} \tilde{u}_{p, j} \widetilde{T}_{p}}{\partial x_{j}} & =-\frac{\tilde{n}_{p} C_{p}}{\tau_{\theta}}\left(\widetilde{T}_{p}-T_{f}\right)-\frac{\partial \tilde{n}_{p} C_{p} \delta \Theta_{p, j}}{\partial x_{j}},
\end{aligned}
$$

where $\tilde{n}_{p}$ is the mesoscopic particle number density and $u_{f}$ and $T_{f}$ the fluid velocity and temperature, respectively. There are two unclosed terms in these equations corresponding to the RUM velocity stress tensor, $\delta R_{p, i j}$, and the RUM heat flux, $\delta \Theta_{p, j}$, defined as:

$$
\begin{aligned}
\delta R_{p, i j} & =\left\langle\delta u_{p, i} \delta u_{p, j} \mid \mathcal{H}_{f}\right\rangle, \\
\delta \Theta_{p, j} & =\left\langle\delta T_{p} \delta u_{p, j} \mid \mathcal{H}_{f}\right\rangle .
\end{aligned}
$$

\subsubsection{Models for the RUM}

The RUM velocity stress tensor is decomposed into its spherical and deviatoric parts as

$$
\delta R_{p, i j}=\delta R_{p, i j}^{*}+\frac{2}{3} \delta \theta_{p} \delta_{i j}
$$

where $\delta \theta_{p}=1 / 2 \delta R_{p, k k}$ is the RUM kinetic energy. 
Recently, Masi et al. [24] proposed a viscosity-like model for the deviatoric part $\delta R_{p, i j}^{*}$, assuming the axisymmetry of tensors, their alignment and a onecomponent limit state:

$$
\delta R_{p, i j}^{*}=\operatorname{sign}\left(I I I_{s}\right)\left(\frac{2}{3}\right)^{1 / 2} 2 \delta \theta_{p} \frac{S_{p, i j}^{*}}{S},
$$

where $S_{p, i j}^{*}$ is the deviatoric part of the mesoscopic particle rate-of-strain tensor, $S$ the square root of its second invariant, and $I I I_{s}$ its third invariant. This RUM model, denoted AXISY-C, was found to improve significantly the prediction of RUM stresses in comparison with the previous model [25, 24, 13]. The main difference is that it now accounts for positive and negative local viscosity and use a more appropriate timescale $\mathcal{F}\left(S^{-1}\right)$, predicting a better mean dissipation.

Then, a transport equation is solved for the RUM kinetic energy:

$$
\frac{\partial \tilde{n}_{p} \delta \theta_{p}}{\partial t}+\frac{\partial \tilde{n}_{p} \tilde{u}_{p, j} \delta \theta_{p}}{\partial x_{j}}=-2 \frac{\tilde{n}_{p}}{\tau_{p}} \delta \theta_{p}-\tilde{n}_{p} \delta R_{p, i j} \frac{\partial \tilde{u}_{p, i}}{\partial x_{j}}-\frac{1}{2} \frac{\partial \tilde{n}_{p} \delta Q_{p, i i j}}{\partial x_{j}} .
$$

The third-order velocity correlation $\delta Q_{p, i j k}=\left\langle\delta u_{p, i} \delta u_{p, j} \delta u_{p, k} \mid \mathcal{H}_{f}\right\rangle$ in Eq. 18 is modeled as suggested by Kaufmann et al. [26]:

$$
\begin{aligned}
\delta Q_{p, i i j} & =-2 \kappa_{p, R U M} \frac{\partial \delta \theta_{p}}{\partial x_{j}}, \\
\kappa_{p, R U M} & =\frac{5 \tau_{p}}{3} \delta \theta_{p} .
\end{aligned}
$$

With this, Eq. 12 is closed so the last contribution to model is the RUM heat flux $\delta \Theta_{p}$ in Eq. 13. The present work being an a posteriori evaluation of the influence of RUM heat fluxes on an academic configuration, it was decided to use as little additional modeling as possible for $\delta \Theta_{p}$. Consequently, we opted for a resolution of the conservation equations for $\delta \Theta_{p}$ derived by Masi [14]: $\frac{\partial \tilde{n}_{p} C_{p} \delta \Theta_{p, i}}{\partial t}+\frac{\partial \tilde{n}_{p} C_{p} \tilde{u}_{p, j} \delta \Theta_{p, i}}{\partial x_{j}}=-\tilde{n}_{p} C_{p}\left(\frac{1}{\tau_{p}}+\frac{1}{\tau_{\theta}}\right) \delta \Theta_{p, i}-\tilde{n}_{p} C_{p} \delta \Theta_{p, j} \frac{\partial \tilde{u}_{p, i}}{\partial x_{j}}$ 


$$
-\tilde{n}_{p} C_{p} \delta R_{p, i j} \frac{\partial \widetilde{T}_{p}}{\partial x_{j}}-\frac{\partial \tilde{n}_{p} \delta \Delta_{p, i j}}{\partial x_{j}},
$$

with the only assumption that the third-order contribution $\delta \Delta_{p, i j}=\left\langle\delta u_{p, i} \delta u_{p, j} \delta T_{p} \mid \mathcal{H}_{f}\right\rangle$ could be neglected. This last assumption is solely based on pragmatism as we do not yet have models available for this term.

\section{Configuration and boundary conditions}

\subsection{Computational domain}

The configuration (Fig. 1) is a two-dimensional cold jet, laden with solid particles and surrounded by a hot co-flow. The jet width, $L_{r e f}=0.7910^{-2} \mathrm{~m}$, is used throughout the paper for normalization. The streamwise ( $x$-axis) extent of the computational domain is $L_{x}=12 L_{\text {ref }}$ and its cross-stream (y-direction) dimension is $L_{y}=6 L_{r e f}$. The carrier gas is composed of pure air (density $\rho_{f}$ and kinematic viscosity $\mu_{f}$ ) at a mean pressure $P_{r e f}$. The parameters common to all computations (velocity $u$, temperature $T$ and mass loading $\alpha$ ) are presented in Tab. 1, where the superscript ' $j$ ' (respectively ' $c$ ') denotes jet (respectively co-flow) properties and the subscript ' $f$ (respectively ' $p$ ') denotes carrier fluid (respectively particles) properties. With these parameters, the Reynolds number based on the momentum thickness is $\operatorname{Re}_{\theta}=\rho_{f} \delta_{\theta} \Delta U / \mu_{f}=125$, where $\Delta U=u_{f}^{j}-u_{f}^{c}$ is the initial velocity difference between the two gaseous streams. Finally, one defines a convective time $t_{c}=12 L_{r e f} / \Delta U$ so that the results can be presented as a function of the normalized variables: $t^{\star}=t / t_{c}, x^{\star}=x / L_{\text {ref }}$ and $y^{\star}=y / L_{r e f}$. It should be pointed out that the two-dimensional character of the configuration is not limiting for the MEF: the RUM formulation was tested successfully in a 


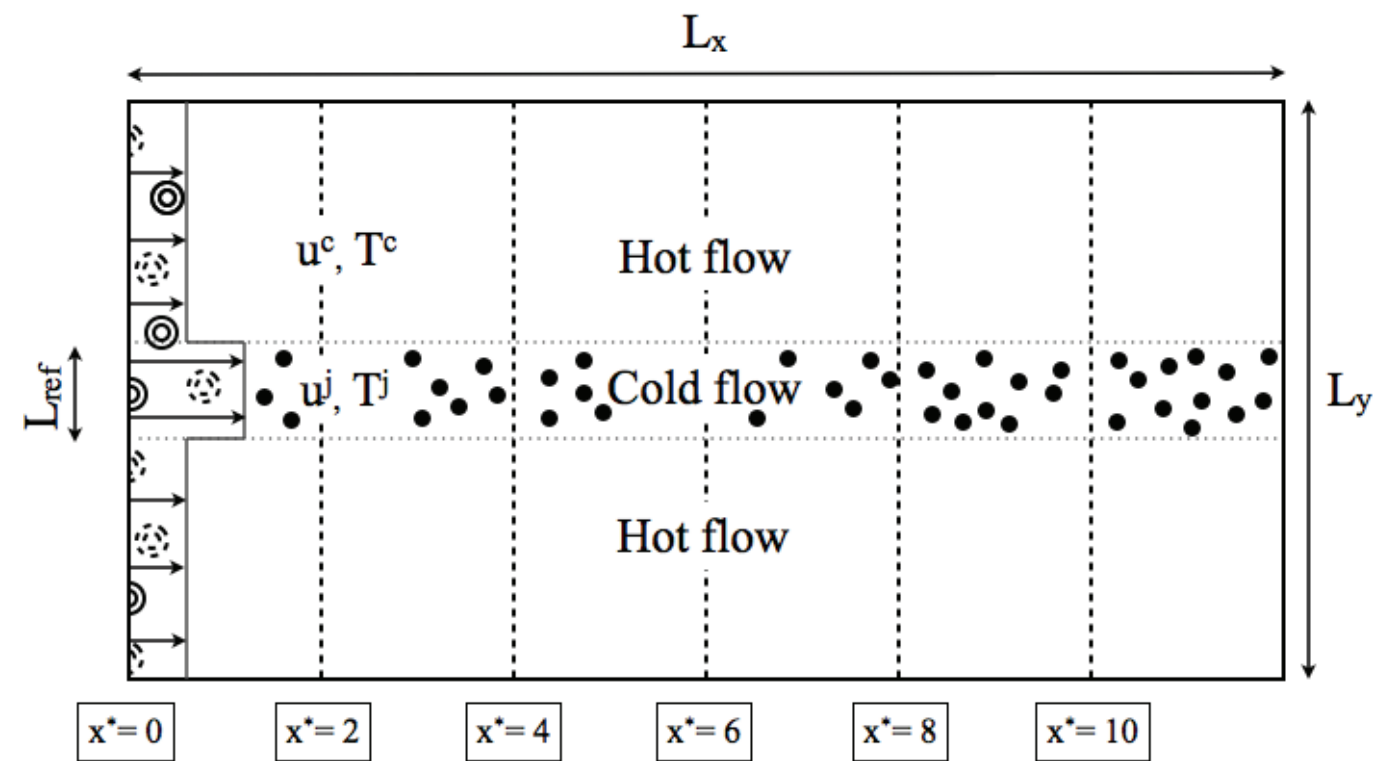

Figure 1: Scheme of the two-dimensional non-isothermal jet. The cold jet at the center is embedded with cold particles while the co-flow is a hot gas void of particles. The vertical dashed lines represent the location of the transverse cuts for the analysis.

three-dimensional test case by Masi et. al [15]. Here, to focus on the effects of heat transfer, using two-dimensional simulations is sufficient to investigate the effects of the RHS terms in Eq. 13.

\subsection{Mesh and boundary conditions}

Both solvers use a cartesian mesh with 1024 cells in $x$ direction and 512 cells in $y$ direction. Mesh independency was thoroughly checked with both solvers: the results presented here are the same with half the current resolution in both directions. Because Asphodele is a low-Mach solver while AVBP is fully compressible, the treatments of inlet and outlet boundary conditions differ: Asphodele uses Dirichlet conditions while AVBP uses characteristic 


\begin{tabular}{cccc}
\hline$L_{\text {ref }}[\mathrm{m}]$ & $\operatorname{Re}_{\theta}$ & $\rho_{f}\left[\mathrm{~kg} \cdot \mathrm{m}^{-3}\right]$ & $\mu_{f}\left[\mathrm{~kg} \cdot \mathrm{m}^{-1} \cdot \mathrm{s}^{-1}\right]$ \\
$0.79 \cdot 10^{-2}$ & 125 & 1.177 & $1.86 \cdot 10^{-5}$ \\
\hline$\delta_{\theta}$ & $T_{f}^{j}, T_{f}^{c}[K]$ & $u_{f}^{j}, u_{f}^{c}\left[\mathrm{~m} \cdot \mathrm{s}^{-1}\right]$ & $T_{p}^{j}[\mathrm{~K}], u_{p}^{j}\left[\mathrm{~m} \cdot \mathrm{s}^{-1}\right], \alpha_{p}^{j}$ \\
$L_{\text {ref }} / 40$ & 300,600 & 20,10 & $300,20,10^{-2}$ \\
\hline
\end{tabular}

Table 1: Parameters for the simulations.

boundary conditions [27] and their recent extension accounting for transverse terms at outlets [28]. Finally, the upper and lower boundary conditions are treated as symmetries.

Axial velocity and temperature of the carrier and dispersed phase, as well as particle volume fraction, are injected with the general hyperbolic profile

$$
\begin{array}{r}
\phi(y)=\phi^{c}+f(y)\left(\phi^{j}-\phi^{c}\right) \\
f(y)=\frac{1}{2}\left(1+\tanh \frac{L_{r e f} / 2-|y|}{2 \delta_{\theta}}\right),
\end{array}
$$

where $\phi^{j}$ and $\phi^{c}$ denote the considered quantity in the jet and co-flow, respectively. All quantities are injected with the same profile in both codes.

\subsection{Turbulence injection}

For a meaningful comparison of the dispersed-phase properties in the two solvers, it is mandatory that they both compute the same carrier phase. It is necessary that the statistics of the carrier to be identical but we have chosen a more conservative approach by imposing exactly the same inlet velocity signal, including the turbulent fluctuations. Mean inlet conditions are presented in Sec. 3.2 and in order to favor the destabilization of the jet, a turbulent velocity fluctuation was added to the mean flow following 
the procedure proposed by Celik and Kraichan [29, 30]. In this particular method, a number of random modes with an average spectrum corresponding to the Passot-Pouquet spectrum are added to the mean velocity. The PassotPouquet spectrum is defined by its most energetic length scale set at $L_{r e f} / 3$ and a turbulent intensity of $2.5 \%$ of the velocity profile. The equivalence of the velocity signals was ensure by imposing in Asphodele the random modes from AVBP.

AVBP being a compressible code, it is only in the absence of acoustic perturbation that the velocity signals can be expected to be identical. Fig. 2 compares the axial and transverse velocities at $x^{\star}=0$ in the middle of the jet for both codes. The agreement is excellent with minor discrepancies caused by the presence of acoustic waves in AVBP.

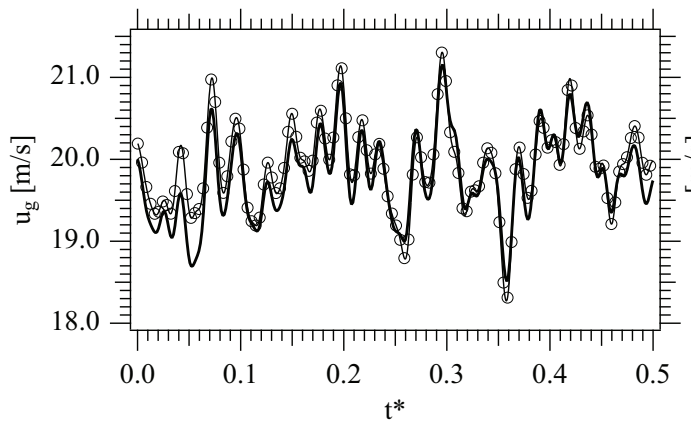

(a)

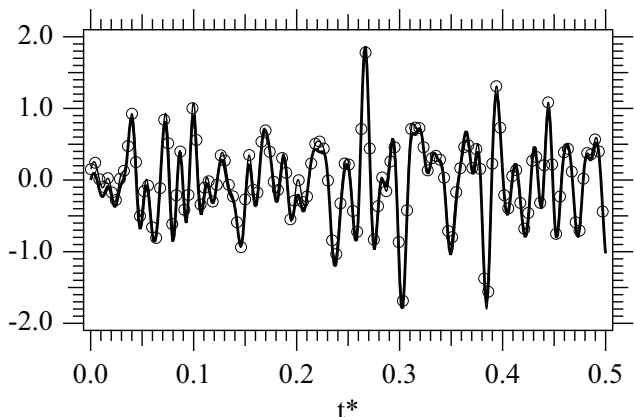

(b)

Figure 2: Comparison of AVBP (—) and Asphodele ( $\bigcirc-$ ) gaseous velocities as a function of $t^{\star}$ at $x^{\star}=0$ in the middle of the jet. (a) Axial velocity, (b) Transverse velocity.

\subsection{Dispersed phase characteristics}

The dynamics of the dispersed phase is governed by the Stokes number, St, while its temperature is driven by the thermal Stokes number $\mathrm{St}_{\theta}$. Fol- 
lowing Eq. 3 one has to prescribe a particle diameter $d_{p}$ and density $\rho_{p}$. For this particular flow, with our choice of fluid characteristic time (Eq. 10), it was found that significant preferential concentration (c.f Fig. 4) was produced for $\mathrm{St}=2$, corresponding to $d_{p}=11.5 \cdot 10^{-6} \mathrm{~m}$ and $\rho_{p}=1999.2 \mathrm{~kg} \cdot \mathrm{m}^{-3}$. All simulations are carried out for this Stokes number, meaning that the dynamics of the jet is fixed for all cases. $\mathrm{St}_{\theta}$ is modulated by changing the particle heat capacity (c.f Eq. 5) to explore its influence on the temperature of the particles. Three values for $\mathrm{St}_{\theta}$ are considered: from thermal tracers $\left(\mathrm{St}_{\theta}=0.2\right)$ to 'thermally ballistic' particles $\left(\mathrm{St}_{\theta}=8\right)$ with an intermediate value $\left(\mathrm{St}_{\theta}=2\right)$ maximizing the effect of $\mathrm{RUM}$ heat fluxes. These parameters are summarized in Tab. 2.

\begin{tabular}{ccc}
\hline$d_{p}[\mathrm{~m}]$ & $\rho_{p}\left[\mathrm{~kg} . \mathrm{m}^{-3}\right]$ & $\mathrm{Pr}$ \\
$11.5 \cdot 10^{-6}$ & 1999.2 & 0.7194 \\
\hline$\alpha=C_{p} / C_{f}$ & $\mathrm{St}$ & $\mathrm{St}_{\theta}$ \\
0.093 & 2 & 0.2 \\
0.93 & - & 2 \\
3.7 & - & 8 \\
\hline
\end{tabular}

Table 2: Characteristics of the three dispersed-phase simulation

Mesoscopic Eulerian quantities are obtained from the Lagrangian simulations by projection on the Eulerian grid. It is possible to circumvent the intrinsic filtering and statistical error of the projection method by using a sufficient number of particles and a well chosen projector [31]. Following these recommendations, ten particles per cell are injected. This procedure 
allows for a direct validation of the Eulerian simulations conducted in AVBP.

Hereinafter, all the statistics of the dispersed-phase used for the validation procedure are conditional averages. The conditional-average operator of a mesoscopic quantity, $<\tilde{\phi}>_{p}$ is defined as

$$
<\tilde{\phi}>_{p}=\frac{\left\{\tilde{n}_{p} \tilde{\phi}\right\}}{\left\{\tilde{n}_{p}\right\}},
$$

where $\{\bullet\}$ is the time-average operator and $\tilde{n}_{p}$ the mesoscopic number density. For the sake of simplicity, the brackets are dropped in the rest of the paper.

\section{Results and analysis}

\subsection{Carrier phase}

As described in Sec. 3.3, it is mandatory that the gaseous phase in both solvers be identical, which is verified in this section. For the sake of compactness, only two statistics, most relevant for the present study are presented: the kinetic energy and rms of the temperature. These variables are important for the preferential concentration and temperature dispersion of the particles but all other gaseous variables compare accordingly between the two solvers.

The kinetic energy of the gas along six transverse cuts of the domain is presented in Fig. 3(a). Mixing layers at the edges of the jet spread with a slight preference into the low-speed streams, which is consistent with theory [32]. The maximum of the kinetic energy increases with $x^{\star}$, first rapidly from the inlet to $x^{\star}=6$ and then more slowly. At $x^{\star}=6$, the kinetic energy at the center of the jet, begins to increase. The agreement between the two solvers (AVBP and Asphodele) is excellent. Then, the rms of gas 
temperature is shown Fig. 3(b). As for the kinetic energy, two regions can be distinguished: for $x^{\star}<6$ the level of rms increases in the outskirts of the jet while for $x^{\star}>6$ the center of the jet is contaminated and the maximum of rms remains roughly constant. Again, the agreement between the two solvers is excellent, which allows for meaningful analysis of the dispersed phase.

\subsection{Dynamics of the dispersed phase}

Since the three runs of Tab. 2 differ only through the thermal Stokes number, the particle positions and velocity fields are the same for the three runs. A qualitative comparison of the particle field at $t^{\star}=2$ is shown in Fig. 4. As observed in other configurations [33], particles concentrate in regions of high shear and low vorticity. The qualitative agreement between the two codes is remarkable. In particular, thanks to the identical turbulent velocity at the inlet, both fields show the same features at the same location.

For a quantitative validation, the time average of the volume fraction over transverse cuts is presented in Fig. 5(a). The agreement is excellent between the Eulerian and Lagrangian simulations. For a validation of the dynamics of the dispersed phase in the Eulerian solver, the time-averaged mesoscopic turbulent kinetic energy, $\tilde{q}_{p}^{2}=1 / 2<\tilde{u}_{p, i}^{\prime} \tilde{u}_{p, i}^{\prime}>_{p}$, is displayed in Fig. 5(b). At the inlet boundary condition, there is no agitation in the dispersed phase so that $\tilde{q}_{p}^{2}=0$. The transfer of turbulent kinetic energy from the carrier yields an increase in $\tilde{q}_{p}^{2}$, first on the edges, eventually spreading to the entire jet. The level of fluctuation in the dispersed phase reaches third of the fluid kinetic energy, displayed in Fig. 3(a). Such levels, as well as the strong preferential concentration observed in the jet, are consistent with the Stokes number of 
the particles. The details of the mean and rms of the velocity components are not presented here but the agreement between the two solvers is similar. The comparison of the dynamics of the dispersed phase between the Eulerian solver and the Lagrangian reference is excellent, which is a validation of the AXISY-C model (Eq. 17) and now allows for a detailed investigation of heat transfer to the dispersed phase.

\subsection{Influence of thermal inertia: analysis of Lagrangian simulations}

As presented in Tab. 2, the dynamical Stokes number is kept constant while the thermal Stokes number $\mathrm{St}_{\theta}$ is varied. In this section, only the Lagrangian results are presented for an analysis of the influence of $\mathrm{St}_{\theta}$, while Sec. 4.4 is devoted to the validation of the Eulerian simulations and the study of the influence of the RUM heat transfer.

The time-averaged mesocopic temperature, $\widetilde{T}_{p}$, and its rms, $\widetilde{T}_{p}^{r m s}$, are shown in Fig. 6 for the three thermal inertia. The corresponding quantity for the gaseous phase is shown in order to quantify the deviation between both phases. The thermal Stokes number $\mathrm{St}_{\theta}$ has a strong impact, both on the mean (Fig. 6(a)) and rms (Fig. 6(b)) of the particle temperature. As expected, for low values of $\mathrm{St}_{\theta}$, the temperature of the particles follows that of the gas yielding identical mean and rms. As the characteristic thermal time of the particles is increased, particles keep the memory of their temperature at injection. The fluctuations are affected accordingly, levels falling down to one fourth of the gas temperature rms for the higher thermal Stokes number. In the early development of the jet, only the particles with the lowest thermal inertia have the same statistics as the gas. However, as the jet evolves, the intermediate value of $\mathrm{St}_{\theta}$ gets closer to the gaseous phase 
while the most inertial particles keep the memory of their initial state and show drastically different statistics. These observations are consistent with the study of temporal evolution of particle temperature variance in decaying non-isothermal homogeneous turbulent configuration [8].

\subsection{Influence of thermal inertia: validation of the Eulerian simulations}

The objective of this section is to validate the Eulerian simulations by comparing them to the Lagrangian reference. The influence of the RUM heat flux on the statistics of the dispersed phase is also analyzed.

Figure 7 presents the mean and rms of particle mesoscopic temperature at $x^{\star}=6$ for the three values of $\mathrm{St}_{\theta}$. First, the Eulerian simulations are carried out without the RUM heat flux term (second term in the r.h.s of Eq. 13). With this simplification, there is no direct coupling between the RUM and the heat transfer to the particles. Then the simulations are conducted with the RUM heat flux term and the resolution of its transport equation (Eq. 21). As shown in Fig. 7(a), at the lowest thermal inertia $\left(\mathrm{St}_{\theta}=0.2\right)$, the Eulerian simulation recovers the Lagrangian result for the time-averaged mesoscopic temperature. However, as $\mathrm{St}_{\theta}$ is increased, the Eulerian results depart from their Lagrangian counterpart at the edges of the jet. Accounting for the RUM heat flux marginally reduces the discrepancy, but overall, it seems that the mean mesoscopic temperature is mildly sensitive to the RUM heat flux term. It should be pointed out that the differences occur in a region of very

small mass loading (c.f. Fig. 5(a)). Particle temperature fluctuations, $\widetilde{T}_{p}^{r m s}$, are presented in Fig. 7(b). For low and intermediate values of $\mathrm{St}_{\theta}$, neglecting the RUM heat flux leads to a deviation of the order of $10 \mathrm{~K}$ in the temperature fluctuations. It is important to note that while the fluctuations are 
underestimated at $\mathrm{St}_{\theta}=0.2$, they are overestimated at $\mathrm{St}_{\theta}=2$. Accounting for RUM heat fluxes allows to recover the correct fluctuation levels. For the case with a very large thermal inertia $\left(\mathrm{St}_{\theta}=8\right)$, the comparison with the lagrangian reference is not favorable, especially at the edges of the jet. Accounting for RUM heat fluxes unfortunately does not yield measurable improvement. For very large values of the thermal inertia, it is likely that the particles are quite far from equilibrium, which is not favorable to the present Eulerian description. This could explain the lack of precision of the present results at $\mathrm{St}_{\theta}=8$.

\section{Conclusions}

The simulation of a two-dimensional turbulent non-isothermal jet laden with solid particles has been carried out using Eulerian-Eulerian and EulerianLagrangian approaches, in two different solvers. A special care has been taken to implement the same injection of turbulence in the two solvers so that time-wise comparison could be performed. The carrier-phase was compared between both codes and a perfect agreement was found.

Cold solid particles were then injected at regime of Stokes number where significant preferential concentration occurs. Again, a very good agreement was found between Eulerian and Lagrangian approaches allowing for a detailed scrutiny of heat transfer.

The influence of the thermal inertia of particles on their temperature was then investigated. The mean and rms of particle temperature showed a strong dependance on the thermal Stokes number. At low, thermal inertia, both mean and rms of particle temperature follow that of the fluid. At high 
thermal inertia, particles keep the memory of their injection temperature so that their statistics differ from that of the surrounding fluid.

Finally, the influence of the RUM heat fluxes in the Mesoscopic Eulerian Formalism was investigated. The mean temperature is satisfactorily predicted by the MEF, comparing to the Lagrangian reference. Under the conditions of the present study, the RUM heat fluxes have a marginal influence on the mean particle temperature. But a significant impact was observed on the magnitude of particle temperature fluctuations. Neglecting the RUM heat fluxes leads to erroneous results while the Lagrangian statistics are recovered when they are accounted for in the regimes of low to moderate thermal Stokes number. However, for particles with a very large thermal inertia $\left(\mathrm{St}_{\theta}=8\right)$, the predictions of the temperature fluctuations deteriorate, even when RUM heat fluxes are accounted for.

\section{Aknowlegments}

The authors acknowledge the financial support from ANR SIGLE grant No ANR-07-PDIT-002-01.

This work was granted access to the high-performance computing resources of CINES under the allocation 2010-x2010026319 made by Grand Equipement National de Calcul Intensif.

The authors would also like to thank Dr. E. Masi and Prof. O. Simonin for fruitful discussions. 


\section{References}

[1] T. Lieuwen, B. Zinn, The role of equivalence ratio oscillations in driving combustion instabilities in low NOx gas turbines, Proc. Combust. Inst. 27 (1998) 1809-1816.

[2] H. Murat Altay, R. L. Speth, D. E. Hudgins, A. F. Ghoniem, The impact of equivalence ratio oscillations on combustion dynamics in a backwardfacing step combustor, Combust. Flame 156 (2009) 2106-2116.

[3] K. Kim, J. Lee, B. Quay, D. Santavicca, Response of partially premixed flames to acoustic velocity and equivalence ratio perturbations, Combust. Flame 157 (2010) 1731-1744.

[4] L. Yarin, G. Hetsroni, Turbulence intensity in dilute two-phase flows-2 temperature fluctuations in particle-laden dilute flows, Int. J. Multiphase Flow 20 (1994) $17-25$.

[5] M. R. Vetrano, S. Gauthier, J. van Beeck, P. Boulet, J.-M. Buchlin, Characterization of a non-isothermal water spray by global rainbow thermometry, Exp. Fluids 40 (2006) 15-22.

[6] Y. Sato, E. Deutsch, O. Simonin, Direct numerical simulations of heat transfer by solid particles suspended in homogeneous isotropic turbulence, Int. J. Heat and Mass Transfer 19 (1998) 187-192.

[7] F. A. Jaberi, Temperature fluctuations in particle-laden homogeneous turbulent flows, Int. J. Heat and Mass Transfer 41 (1998) 4081 - 4093. 
[8] F. A. Jaberi, F. Mashayek, Temperature decay in two-phase turbulent flows, Int. J. Heat and Mass Transfer 43 (2000) 993 - 1005.

[9] B. Shotorban, F. Mashayek, R. V. R. Pandya, Temperature statistics in particle-laden turbulent homogeneous shear flow, International Journal of Multiphase Flow 29 (2003) 1333 - 1353.

[10] J. Ferry, E. Balachandar, Equilibirum eulerian approach for predicting the thermal field of a dispersion of small particles, Int. J. Heat and Mass Transfer 48 (2005) 681-689.

[11] P. Février, O. Simonin, K. Squires, Partitioning of particle velocities in gas-solid turbulent flows into a continuous field and a spatially uncorrelated random distribution: Theoretical formalism and numerical study, J. Fluid Mech. 533 (2005) 1-46.

[12] E. Riber, M. Moreau, O. Simonin, B. Cuenot, Towards large eddy simulation of non-homogeneous particle laden turbulent gas flows using euler-euler approach, in: 11th Workshop on Two-Phase Flow Predictions, Merseburg, Germany.

[13] E. Riber, V. Moureau, M. García., T. Poinsot, O. Simonin, Evaluation of numerical strategies for LES of two-phase reacting flows, J. Comput. Phys. 228 (2009) 539-564.

[14] E. Masi, Theoretical and numerical study of the modeling of unsteady non-isothermal particle-laden turbulent flows by an Eulerian-Eulerian approach, Phd thesis, INP Toulouse, 2010. 
[15] E. Masi, O. Simonin, B. Bédat, The mesoscopic eulerian approach for evaporating droplets interacting with turbulent flows, Flow, Turb. and Combustion 86 (2011) 563-583.

[16] V. Moureau, G. Lartigue, Y. Sommerer, C. Angelberger, O. Colin, T. Poinsot, Numerical methods for unsteady compressible multicomponent reacting flows on fixed and moving grids, J. Comput. Phys. 202 (2005) 710-736.

[17] L. Selle, G. Lartigue, T. Poinsot, R. Koch, K.-U. Schildmacher, W. Krebs, B. Prade, P. Kaufmann, D. Veynante, Compressible largeeddy simulation of turbulent combustion in complex geometry on unstructured meshes, Combust. Flame 137 (2004) 489-505.

[18] J. Reveillon, C. Pera, Z. Bouali, Examples of the potential of dns for the understanding of reactive multiphase flows, Int. J. of Spray and Comb. Dyn. 3 (2011) 65-94.

[19] O. Colin, M. Rudgyard, Development of high-order taylor-galerkin schemes for unsteady calculations, J. Comput. Phys. 162 (2000) 338371.

[20] A. A. Wray, Minimal storage time advanced schemes for spectral methods, J. Comput. Phys. (1990).

[21] M. W. Reeks, On a kinetic equation for the transport of particles in turbulent flows, Phys. Fluids 3 (1991).

[22] W. A. Sirignano, Fuel droplet vaporisation and spray combustion theory, Prog. Energy Comb. Sci. 9 (1983) 291-322. 
[23] S. K. Aggarwal, Relationship between stokes number and intrinsic frequencies in particle-laden flows, AIAA Journal 32 (1994) 1322-1325.

[24] E. Masi, E. Riber, P. Sierra, O. Simonin, L. Gicquel, Modeling the random uncorrelatex velocity stress tensor for unsteady particles eulerian simulation in turbulent flows, in: Proc. 7th Int. Conf. Multiphase Flow, ICFM-2010, Tampa, USA.

[25] A. Kaufmann, Vers la simulation des grandes échelles en formulation Euler/Euler des écoulements réactifs diphasiques, Phd thesis, INP Toulouse, 2004.

[26] A. Kaufmann, M. Moreau, O. Simonin, J. Hélie, Comparison between lagrangian and mesoscopic eulerian modelling approaches for inertial particles suspended in decaying isotropic turbulence, J. Comput. Phys. 227 (2008) 6448-6472.

[27] T. Poinsot, S. Lele, Boundary conditions for direct simulations of compressible viscous flows, J. Comput. Phys. 101 (1992) 104-129.

[28] V. Granet, O. Vermorel, T. Leonard, L. Gicquel, , T. Poinsot, Comparison of nonreflecting outlet boundary conditions for compressible solvers on unstructured grids, Am. Inst. Aeronaut. Astronaut. J. 48 (2010) 2348-2364.

[29] R. Kraichnan, Diffusion by a random velocity field, Phys. Fluids 13 (1970) 22-31.

[30] A. Smirnov, S. Shi, I. Celik, Random flow generation technique for 
large eddy simulations and particle-dynamics modeling, Trans. ASME. J. Fluids Eng. 123 (2001) 359-371.

[31] A. Kaufmann, M. Moreau, O. Simonin, J. Hélie, Comparison between lagrangian and mesoscopic eulerian modelling approaches for inertial particles suspended in decaying isotropic turbulence, J. Comput. Phys. 227 (2008) 6448-6472.

[32] S. B. Pope, Turbulent flows, Cambridge University Press, 2000.

[33] K. D. Squires, J. K. Eaton, Preferential concentration of particles by turbulence, Phys. Fluids 3 (1991) 1169-1178. 


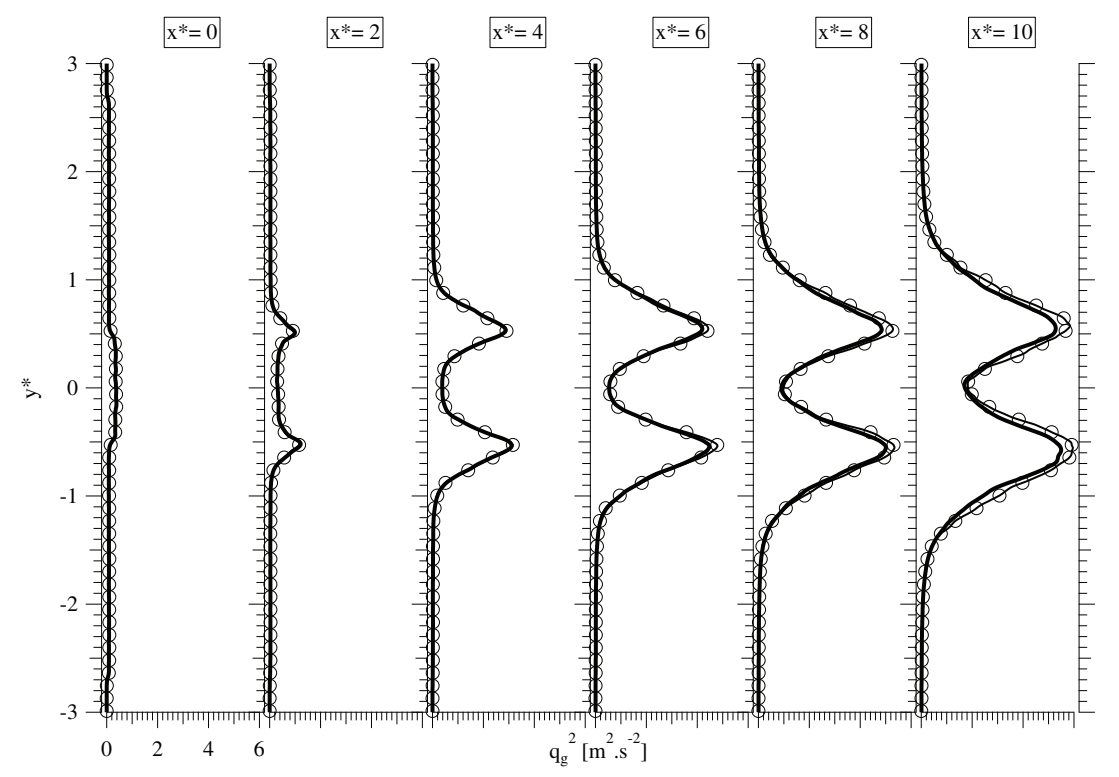

(a)

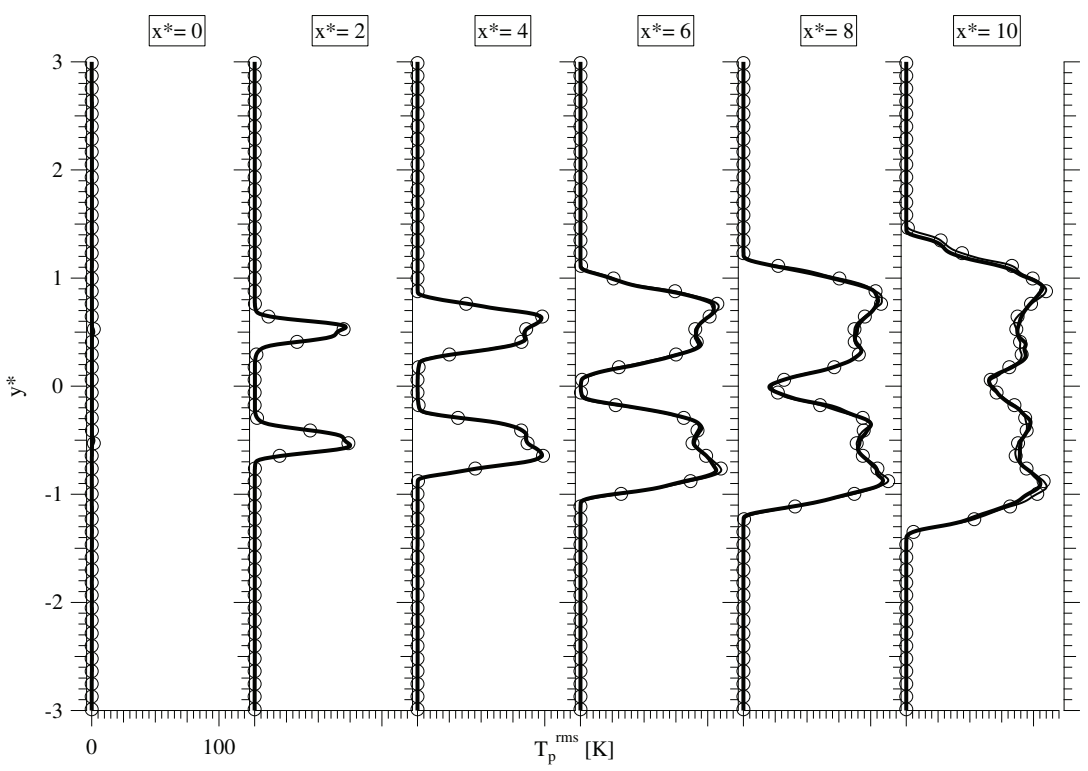

(b)

Figure 3: Comparison between AVBP ) and Asphodele ( $\bigcirc-$ ) at six transverse cuts. (a) Kinetic energy and (b) rms of temperature for the carrier phase. 


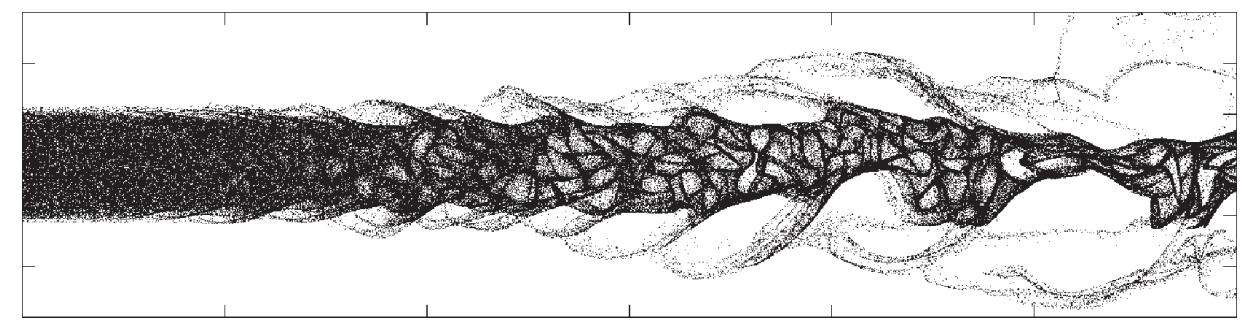

(a)

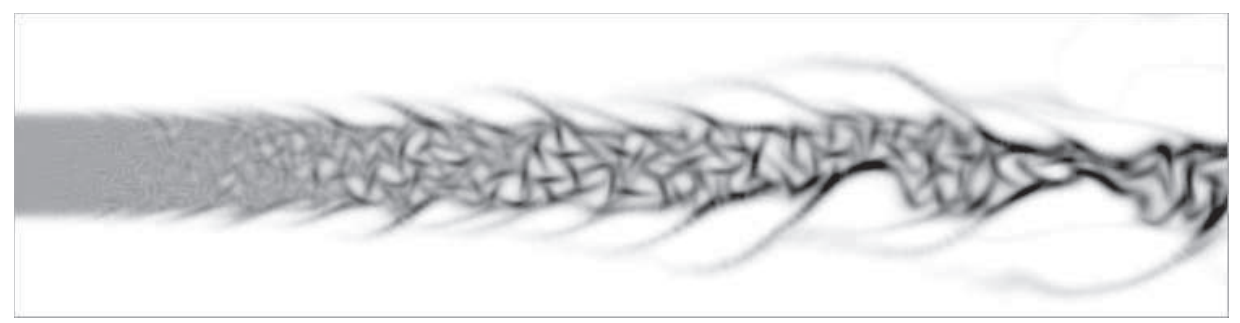

(b)

Figure 4: Particle-laden jet at time $t^{\star}=2$. (a) Lagrangian field of particles. (b) Eulerian particle volume fraction. 


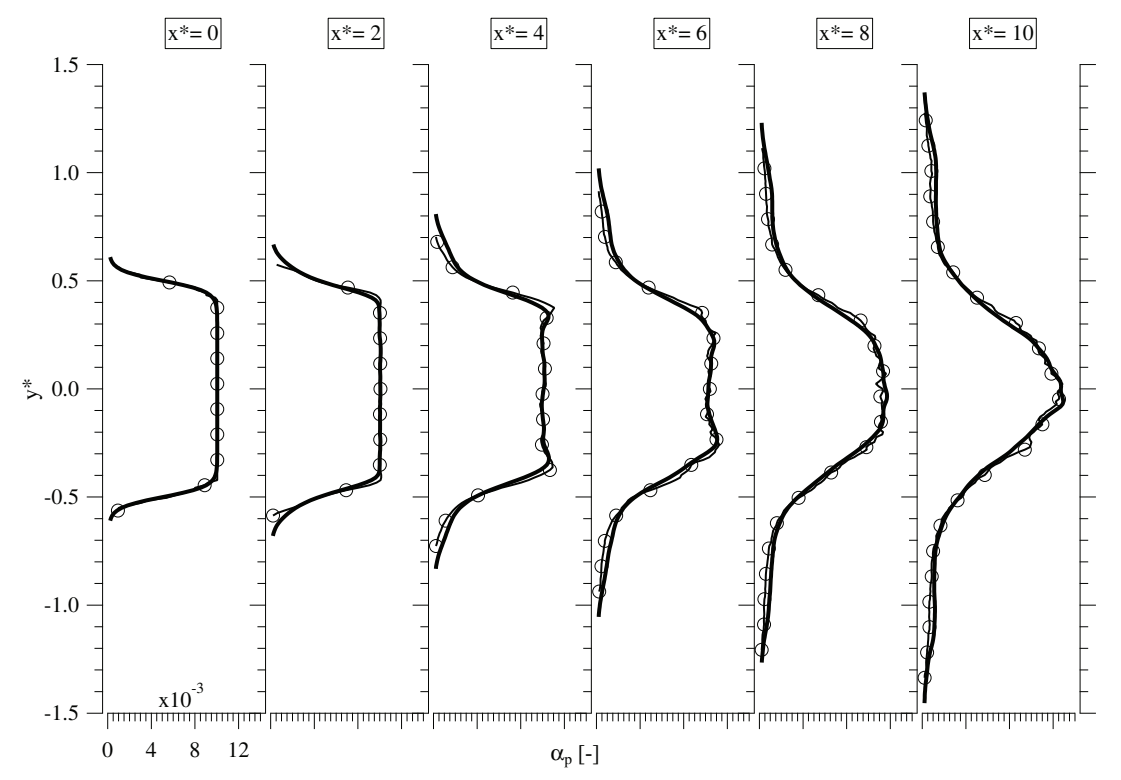

(a)

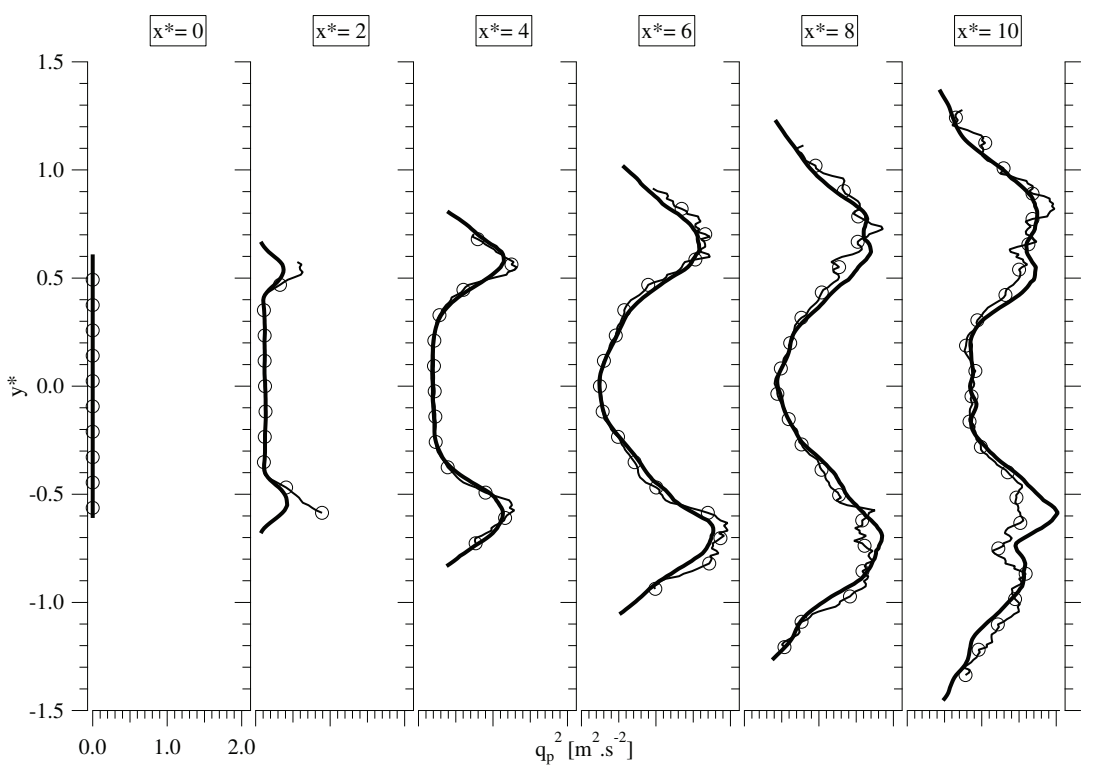

(b)

Figure 5: Comparison between AVBP ) and Asphodele ( $\bigcirc-$ ) at six transverse cuts. (a) particle volume fraction $\alpha_{p}$ and (b) mesoscopic turbulent kinetic energy $\tilde{q}_{p}^{2}$. 


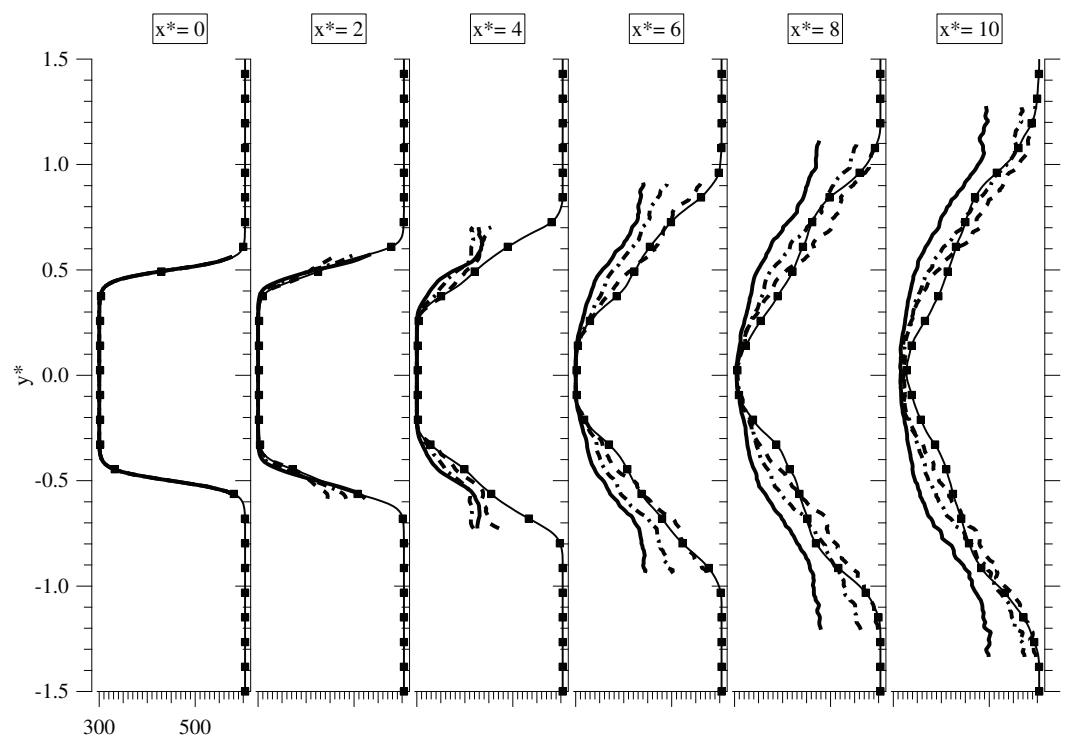

(a) $\widetilde{T}_{p}(\mathrm{~K})$

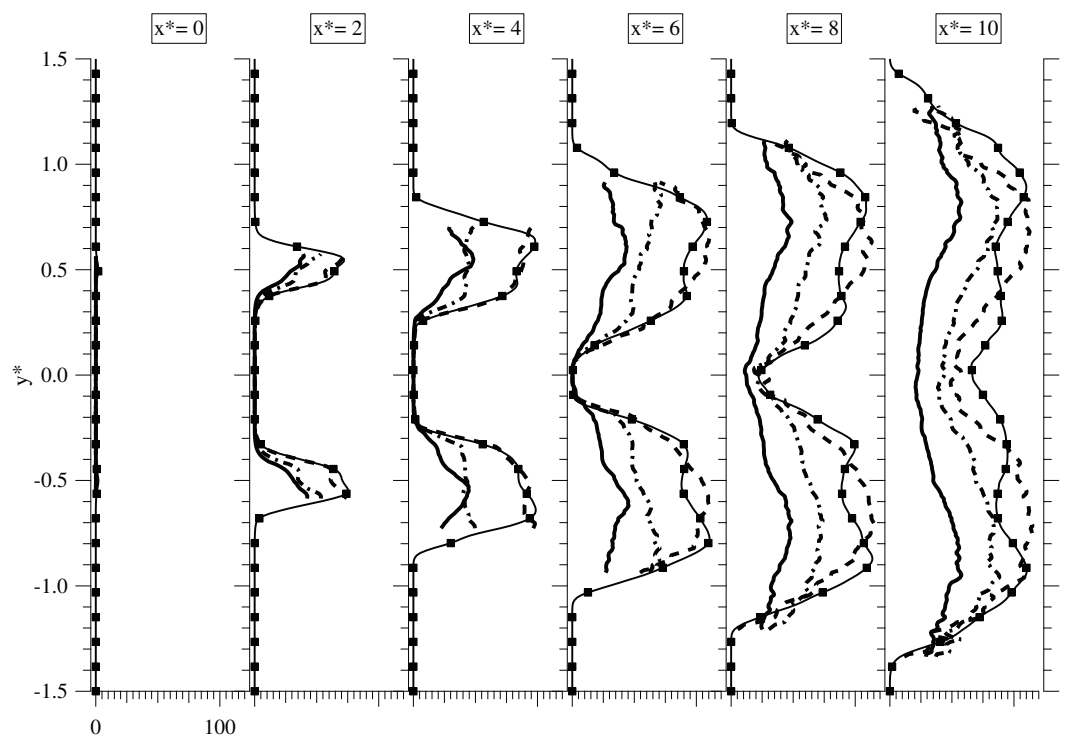

(b) $\widetilde{T}_{p}^{r m s}(\mathrm{~K})$

Figure 6: Lagrangian simulation results: 6(a)time-averaged mesoscopic temperature of the particles, and 6(b) rms ot temperature fluctuations, for different values of the thermal inertia. - $: \mathrm{St}_{\theta}=8 ;-\cdot-\mathrm{St}_{\theta}=2$ and ---- $: \mathrm{St}_{\theta}=0.2$. The corresponding quantity for the carrier fluid is presented for reference: $\longrightarrow-$. 


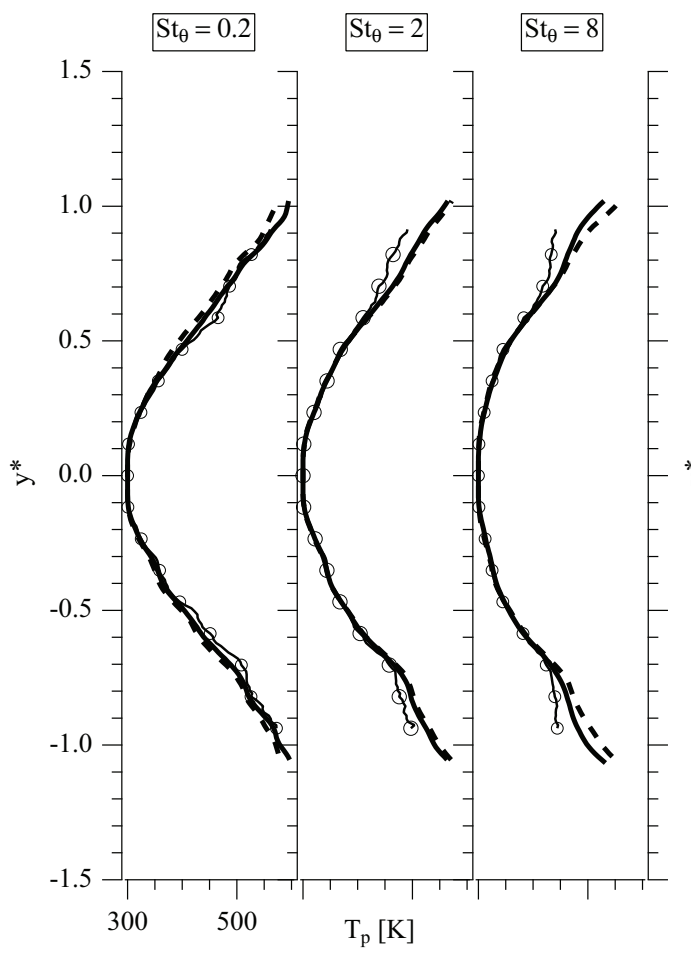

(a)

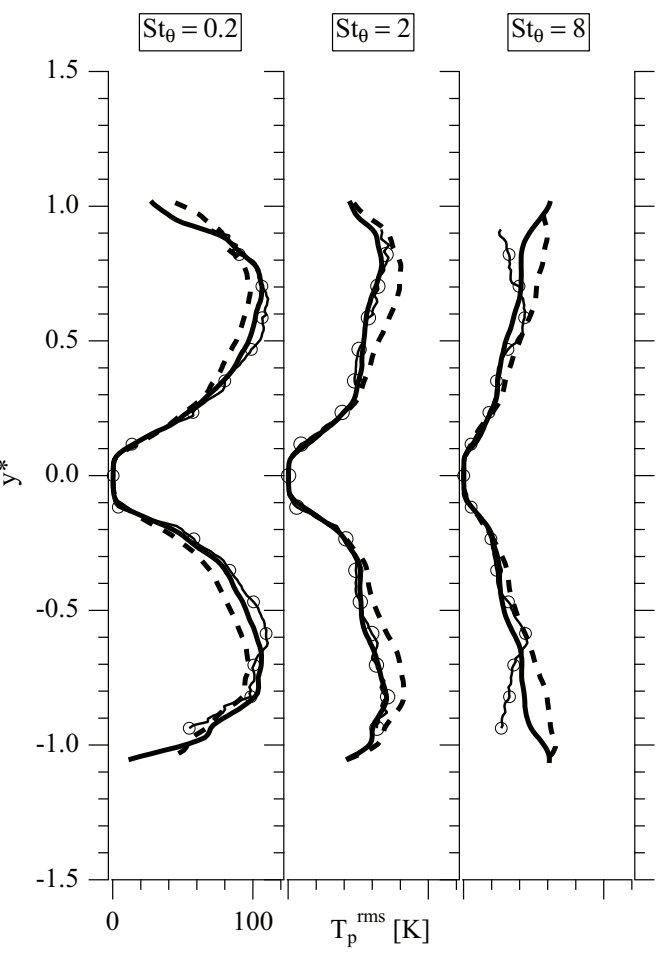

(b)

Figure 7: Comparison of Eulerian and Lagrangian mesoscopic quantities at $x^{\star}=6$. (a) Mean particle temperature $\widetilde{T}_{p}$, (b) rms $\widetilde{T}_{p}^{r m s}$. Influence of the RUM-HF on the Eulerian statistics: - $:$ with RUM-HF, ---- : without RUM-HF, Lagrangian reference (- ). 